\title{
Sex Ambiguity in a 1-year-old Child having Penoscrotal Hypospadias with Bilateral Undescended Testes (Cryptorchidism)
}

\author{
${ }^{1}$ Somanna Prabhakara, ${ }^{2}$ V Sreedhar Reddy, ${ }^{3}$ Kolandaswamy Anbazhagan, ${ }^{4} \mathrm{~J}$ Vineetha Mercy, ${ }^{5}$ Basanth K Reddy
}

\begin{abstract}
Hypospadias with cryptorchidism is not a very common anomaly in infants and preadolescents. However, it is a perplexing situation to ascertain the exact sex of the child and to assign a proper chromosomal and gonadal sex of the child. The testis can be ectopic, incompletely descended, retractile, absent, or atrophic. In hypospadias, the urethral opening is located on the under surface of the penis, anywhere from the glans to the perineum, depending on the severity of the case. It is associated with chordee, patulous glans, and hooded prepuce. The present case is a 1-year-old child having penoscrotal hypospadias and bilateral undescended testes (cryptorchidism) with genital ambiguity. Our aim of the study was to identify the chromosomal sex and to rule out any microdeletion in the $Y$ chromosome of the child by using conventional cytogenetic and polymerase chain reaction (PCR) techniques. We found that the chromosomal sex was male and there was no microdeletion in the $Y$ chromosome.
\end{abstract}

Keywords: Chromosomal sex, Cytogenetic analysis, Genital ambiguity, Prepuce.

How to cite this article: Prabhakara S, Reddy VS, Anbazhagan K, Mercy JV, Reddy BK. Sex Ambiguity in a 1-year-old Child having Penoscrotal Hypospadias with Bilateral Undescended Testes (Cryptorchidism). J Med Sci 2017;3(3):79-81.

Source of support: Nil

Conflict of interest: None

\section{INTRODUCTION}

Hypospadias is one of the most common congenital anomalies, affecting one in 250 to 300 live births. There are higher incidences if first-degree relatives are affected. Up to $26 \%$ of male offspring of an affected father may have hypospadias, and the risk in subsequent siblings is $12 \% .^{1}$ It is more common in male infants who are growth restricted and premature. Other risk factors include

\footnotetext{
${ }^{1}$ Senior Research Scientist and Chairperson, ${ }^{2}$ Professor and Head ${ }^{3}$ Visiting Senior Research Scientist, ${ }^{4}$ Research Assistant ${ }^{5}$ Research Scientist

${ }^{1,3-5}$ Central Research Laboratory, RajaRajeswari Medical College \& Hospital, Bengaluru, Karnataka, India

${ }^{2}$ Department of Urology, RajaRajeswari Medical College \& Hospital, Bengaluru, Karnataka, India

Corresponding Author: Somanna Prabhakara, Senior Research Scientist and Chairperson, Central Research Laboratory RajaRajeswari Medical College \& Hospital, Bengaluru, Karnataka India, Phone: +919482521675, e-mail: prabhakarsom@gmail.com
}

parental subfertility and the frequency of associated anomalies increases with the severity of hypospadias. Cryptorchidism and inguinal hernia are the most commonly associated anomalies. ${ }^{2}$

The surgical approach to repair hypospadias continues to develop, and success rate for single-stage repair approach is $90 \%$. Though there have been many investigations, the causes of hypospadias remain elusive. Genetic, endocrinological, and environmental factors have been implicated. Evidence supporting each factor exists, but no single cause can explain the relatively high incidence of this condition; the cause is probably multifactorial. Hypospadias may be accompanied by different genital malformations. ${ }^{3}$

\section{CASE REPORT}

A 1-year-old child was referred from pediatric outpatient department to Urology Department with the finding of penoscrotal hypospadias and bilateral undescended testes. The phallus was well developed and male type. On examination, the baby appeared well nourished with penis measuring $1.5 \mathrm{~cm}$ long with glans and corporal bodies well developed. Prepuce was not developed on the ventral aspect and there was ventral chordee with penoscrotal hypospadias. Scrotal sac was not well developed and testicles were not felt in scrotal sac, inguinal canal, or per abdomen. There was no other clinical abnormality.

For karyotyping, $3 \mathrm{~mL}$ peripheral blood sample was drawn in to sodium heparinized vacutainer and written consent was obtained. GTG-banding of 72-hour phytohemagglutinin-stimulated cultures of peripheral blood lymphocytes was performed using standard protocols. ${ }^{4,5}$ The preparations were examined using light microscopes. Thirty metaphases were captured and evaluated by using fluorescence microscope (Axioimager A2, Carl Zeiss, Germany) with the help of ISIS software (MetaSystems GmbH, Germany). The karyotyping was reported according to the International System for Human Cytogenetic Nomenclature (2009). Cytogenetic analysis of the proband revealed a normal karyotype (Fig. 1). Further " $\mathrm{Q}$ " banding was performed as per standard protocols, which also confirmed the presence of " $\mathrm{Y}$ " chromosome (Fig. 2). 


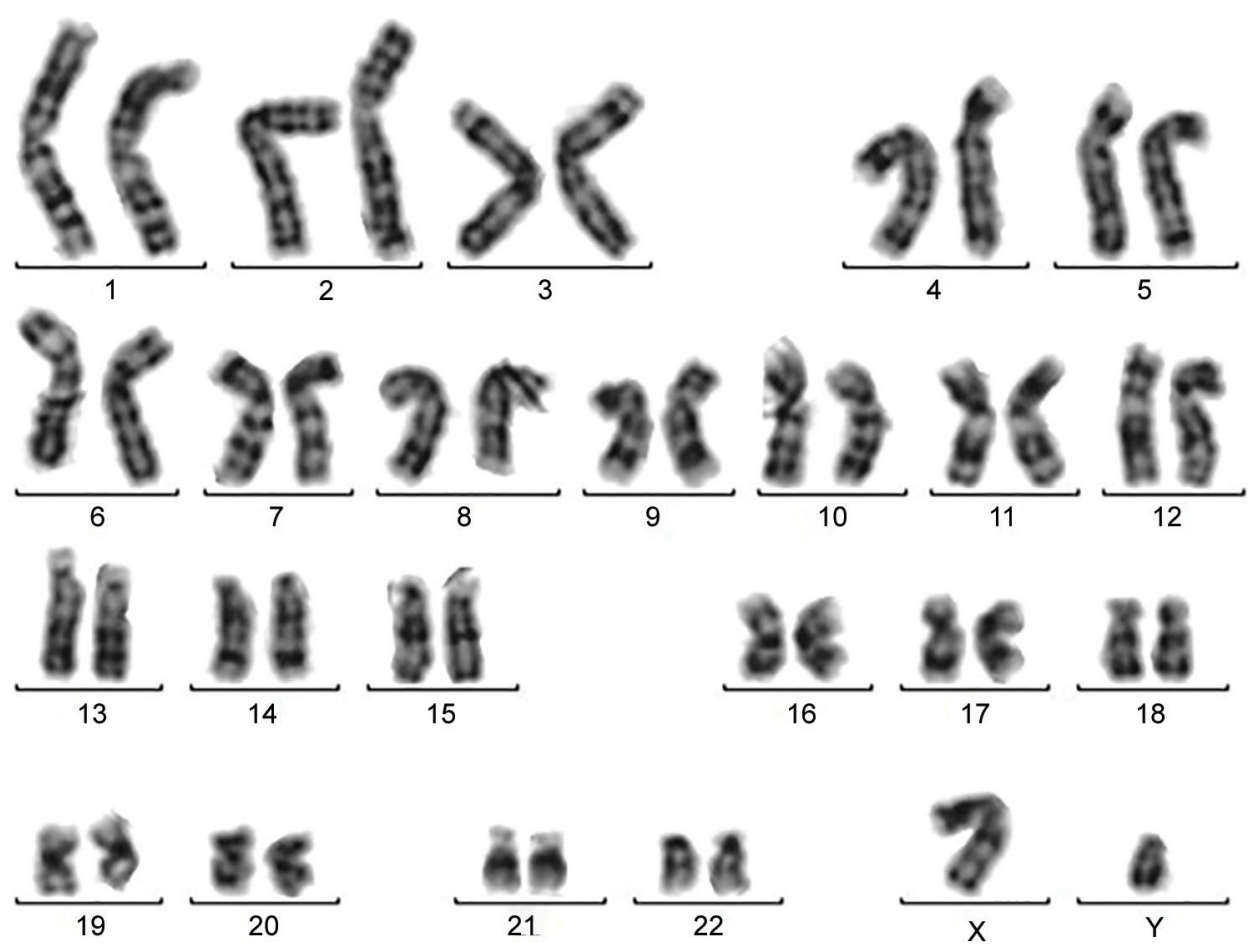

Fig. 1: Karyotype of the patient (normal male; 46, XY)

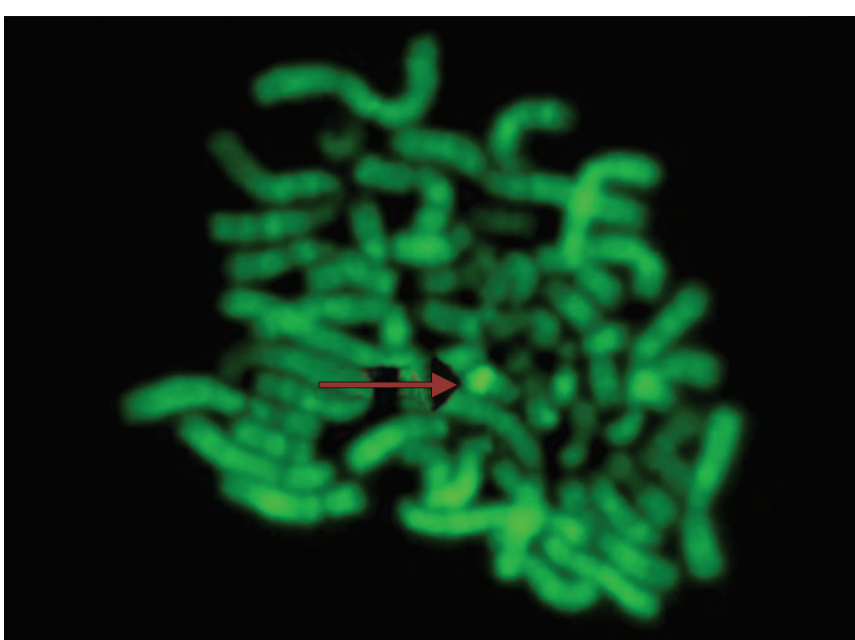

Fig. 2: "Q" banded metaphase of the patient. The arrow mark shows the presence of " $Y$ " chromosome in the patient

The deletion of the azoospermia factor (AZF) region in $Y$ chromosome is thought to be involved in some cases of male infertility causing azoospermia or severe oligozoospermia. ${ }^{6}$ Hence, we performed multiplex PCR for the detection of Y-chromosome microdeletion as per Simoni et al. ${ }^{7}$ Three milliliters of blood was collected in ethylenediaminetetraacetic acid vacutainers from the patient after obtaining written consent. Deoxyribonucleic acid (DNA) was extracted from blood by the standard salting out method. ${ }^{8}$ Five microliters of the dissolved DNA was added to each $25 \mu \mathrm{L}$ PCR reaction that included multiplex primer set as specified by Simoni et al. ${ }^{7}$ The results

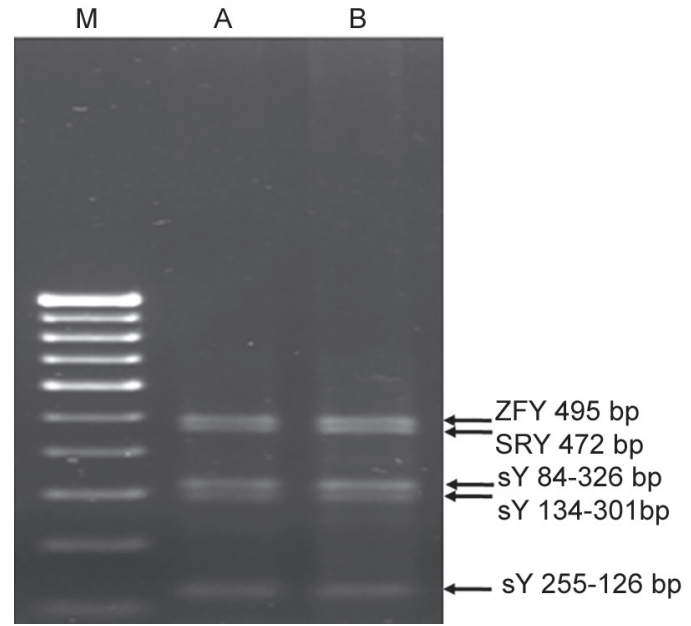

Fig. 3: Multiplex PCR for "Y" chromosome microdeletion. Marker (M) represents the $100 \mathrm{bp}$ DNA ladder. "A" represents the control fertile subject and " $\mathrm{B}$ " represents the patient

indicated that there was no deletion in the $Y$ chromosome (Fig. 3) in the patient, suggesting that the proband is a male and possibly fertile.

\section{DISCUSSION}

The child was referred to us for defining gender with the help of cytogenetic techniques and fertility using Y-chromosome microdeletion analysis. Based on these analyses, the proband with penoscrotal hypospadias and bilateral undescended testes was found to be a male. In addition, there was no microdeletion in the $\mathrm{Y}$ chromosome, indicating possibility of normal fertility. This type 
of penoscrotal hypospadias with bilateral undescended testes is a severe form of genital anomaly and likely to have intersex state. The porband needs further evaluation by magnetic resonance imaging and laparoscopy to detect the presence or absence of gonads in abdomen. Depending on these findings, further steps can be taken to assign the appropriate sex and to take required surgical correction.

Further studies on examination of androgen sensitivity and congenital adrenal hyperplasia are suggested to rule out possibility of intersex gender.

\section{REFERENCES}

1. Bauer SB, Retik AB, Colodny AH. Genetic aspects of hypospadias. Urol Clin North Am 1981 Oct;8(3):559-564.

2. Stojanovic B, Bizic M, Majstorovic M, Kojovic V, Djordjevic M. Penile curvature incidence in hypospadias: can it be determined? Adv Urol 2011 Aug;2011:1-4.

3. Gatti JM, Kirsch AJ, Troyer WA, Perez-Brayfield MR, Smith EA, Scherz HC. Increased incidence of hypospadias in small-for-gestational age infants in a neonatal intensivecare unit. BJU Int 2001 Apr;87(6):548-550.

4. Akbas E, Mutluhan H, Savasoglu K, Soylemez F, Ozturk I, Yazici G. Turner syndrome and 45,X/47,XXX mosaicism. Genet Couns 2009; 20(2):141-146.

5. Venkataswamy E, Nargund AR, Prabhudesai S, Patil GV, Chandra RJ, Veldore VH, Patil S, Verma A, Sahoo R, Ajaikumar BS, et al. An uncommon case of an adult with $\operatorname{del}(5)(q)$ in acute lymphoblastic leukemia. Indian J Hum Genet 2012 Sep-Dec;18(3):349-351.

6. Vogt PH. Human chromosome deletions in Yq11, AZF candidate genes and male infertility: history and update.Mol Hum Reprod 1998 Aug;4(8):739-744.

7. Simoni M, Bakker E, Eurlings MC, Matthijs G, Moro E, Müller CR, Vogt PH. Laboratory guidelines for molecular diagnosis of Y-chromosomal microdeletions.Int J Androl 1999 Oct;22(5):292-299.

8. Kesari A, Mukherjee M, Mittal B. Mutation Analysis in spinal muscular atrophy using allele-specific polymerase chain reaction.Indian J Biochem Biophys 2003 Dec;40(6): 439-441. 